EUROPEAN LABORATORY FOR PARTICLE PHYSICS

CERN PPE/97-138

20 October 1997

\title{
An upper limit on the $\tau$ neutrino mass from three- and five-prong tau decays.
}

\author{
The ALEPH Collaboration*
}

\begin{abstract}
A bound on the tau neutrino mass is established using the data collected from 1991 to 1995 at $\sqrt{s} \simeq m_{Z}$ with the ALEPH detector. Two separate limits are derived by fitting the distribution of visible energy vs invariant mass in $\tau^{-} \rightarrow 2 \pi^{-} \pi^{+} \nu_{\tau}$ and $\tau^{-} \rightarrow$ $3 \pi^{-} 2 \pi^{+}\left(\pi^{0}\right) \nu_{\tau}$ decays. The two results are combined to obtain a $95 \%$ confidence level upper limit of $18.2 \mathrm{MeV} / \mathrm{c}^{2}$ on the mass of the tau neutrino.
\end{abstract}

(submtitted to Zeitschrift für Physik C)

*See the following pages for the list of authors 


\section{The ALEPH Collaboration}

R. Barate, D. Buskulic, D. Decamp, P. Ghez, C. Goy, J.-P. Lees, A. Lucotte, M.-N. Minard, J.-Y. Nief,

B. Pietrzyk

Laboratoire de Physique des Particules (LAPP), $I N^{2} P^{3}$-CNRS, 74019 Annecy-le-Vieux Cedex, France

M.P. Casado, M. Chmeissani, P. Comas, J.M. Crespo, M. Delfino, E. Fernandez, M. Fernandez-Bosman, Ll. Garrido, ${ }^{15}$ A. Juste, M. Martinez, G. Merino, R. Miquel, Ll.M. Mir, C. Padilla, I.C. Park, A. Pascual, J.A. Perlas, I. Riu, F. Sanchez

Institut de Física d'Altes Energies, Universitat Autònoma de Barcelona, 08193 Bellaterra (Barcelona), Spain $^{7}$

A. Colaleo, D. Creanza, M. de Palma, G. Gelao, G. Iaselli, G. Maggi, M. Maggi, N. Marinelli, S. Nuzzo, A. Ranieri, G. Raso, F. Ruggieri, G. Selvaggi, L. Silvestris, P. Tempesta, A. Tricomi, ${ }^{3}$ G. Zito

Dipartimento di Fisica, INFN Sezione di Bari, 70126 Bari, Italy

X. Huang, J. Lin, Q. Ouyang, T. Wang, Y. Xie, R. Xu, S. Xue, J. Zhang, L. Zhang, W. Zhao

Institute of High-Energy Physics, Academia Sinica, Beijing, The People's Republic of China ${ }^{8}$

D. Abbaneo, R. Alemany, U. Becker, P. Bright-Thomas, D. Casper, M. Cattaneo, F. Cerutti,

G. Dissertori, H. Drevermann, R.W. Forty, M. Frank, R. Hagelberg, J.B. Hansen, J. Harvey, P. Janot,

B. Jost, I. Lehraus, P. Mato, A. Minten, L. Moneta, A. Pacheco, J.-F. Pusztaszeri, ${ }^{23}$ F. Ranjard,

L. Rolandi, D. Rousseau, D. Schlatter, M. Schmitt, O. Schneider, W. Tejessy, F. Teubert, I.R. Tomalin,

H. Wachsmuth, A. Wagner ${ }^{20}$

European Laboratory for Particle Physics (CERN), 1211 Geneva 23, Switzerland

Z. Ajaltouni, A. Barrès, C. Boyer, A. Falvard, C. Ferdi, P. Gay, C . Guicheney, P. Henrard, J. Jousset, B. Michel, S. Monteil, J-C. Montret, D. Pallin, P. Perret, F. Podlyski, J. Proriol, P. Rosnet, J.M. Rossignol

Laboratoire de Physique Corpusculaire, Université Blaise Pascal, $I N^{2} P^{3}-C N R S$, Clermont-Ferrand, 63177 Aubière, France

T. Fearnley, J.D. Hansen, J.R. Hansen, P.H. Hansen, B.S. Nilsson, B. Rensch, A. Wäänänen

Niels Bohr Institute, 2100 Copenhagen, Denmark ${ }^{9}$

G. Daskalakis, A. Kyriakis, C. Markou, E. Simopoulou, I. Siotis, A. Vayaki

Nuclear Research Center Demokritos (NRCD), Athens, Greece

A. Blondel, G. Bonneaud, J.C. Brient, P. Bourdon, A. Rougé, M. Rumpf, A. Valassi ${ }^{6}$ M. Verderi, H. Videau

Laboratoire de Physique Nucléaire et des Hautes Energies, Ecole Polytechnique, IN ${ }^{2} P^{3}$-CNRS, 91128

Palaiseau Cedex, France

D.J. Candlin, M.I. Parsons

Department of Physics, University of Edinburgh, Edinburgh EH9 3JZ, United Kingdom ${ }^{10}$

T. Boccali, E. Focardi, G. Parrini, K. Zachariadou

Dipartimento di Fisica, Università di Firenze, INFN Sezione di Firenze, 50125 Firenze, Italy

M. Corden, C. Georgiopoulos, D.E. Jaffe

Supercomputer Computations Research Institute, Florida State University, Tallahassee, FL 323064052, USA ${ }^{13,14}$ 
A. Antonelli, G. Bencivenni, G. Bologna, ${ }^{4}$ F. Bossi, P. Campana, G. Capon, V. Chiarella, G. Felici, P. Laurelli, G. Mannocchi, ${ }^{5}$ F. Murtas, G.P. Murtas, L. Passalacqua, M. Pepe-Altarelli

Laboratori Nazionali dell'INFN (LNF-INFN), 00044 Frascati, Italy

L. Curtis, S.J. Dorris, A.W. Halley, J.G. Lynch, V. O’Shea, C. Raine, J.M. Scarr, K. Smith, P. TeixeiraDias, A.S. Thompson, E. Thomson, F. Thomson

Department of Physics and Astronomy, University of Glasgow, Glasgow G12 8QQ,United Kingdom ${ }^{10}$

O. Buchmüller, S. Dhamotharan, C. Geweniger, G. Graefe, P. Hanke, G. Hansper, V. Hepp, E.E. Kluge, A. Putzer, J. Sommer, K. Tittel, S. Werner, M. Wunsch

Institut für Hochenergiephysik, Universität Heidelberg, 69120 Heidelberg, Fed. Rep. of Germany ${ }^{16}$

R. Beuselinck, D.M. Binnie, W. Cameron, P.J. Dornan, M. Girone, S. Goodsir, E.B. Martin, A. Moutoussi, J. Nash, J.K. Sedgbeer, P. Spagnolo, A.M. Stacey, M.D. Williams

Department of Physics, Imperial College, London SW7 2BZ, United Kingdom ${ }^{10}$

V.M. Ghete, P. Girtler, E. Kneringer, D. Kuhn, G. Rudolph

Institut für Experimentalphysik, Universität Innsbruck, 6020 Innsbruck, Austria ${ }^{18}$

A.P. Betteridge, C.K. Bowdery, P.G. Buck, P. Colrain, G. Crawford, A.J. Finch, F. Foster, G. Hughes, R.W.L. Jones, T. Sloan, M.I. Williams

Department of Physics, University of Lancaster, Lancaster LA1 4YB, United Kingdom ${ }^{10}$

I. Giehl, A.M. Greene, C. Hoffmann, K. Jakobs, K. Kleinknecht, G. Quast, B. Renk, E. Rohne, H.G. Sander, P. van Gemmeren, C. Zeitnitz

Institut für Physik, Universität Mainz, 55099 Mainz, Fed. Rep. of Germany ${ }^{16}$

J.J. Aubert, C. Benchouk, A. Bonissent, G. Bujosa, J. Carr, P. Coyle, C. Diaconu, F. Etienne, O. Leroy, F. Motsch, P. Payre, M. Talby, A. Sadouki, M. Thulasidas, K. Trabelsi

Centre de Physique des Particules, Faculté des Sciences de Luminy, IN ${ }^{2} P^{3}$-CNRS, 13288 Marseille, France

M. Aleppo, M. Antonelli, F. Ragusa

Dipartimento di Fisica, Università di Milano e INFN Sezione di Milano, 20133 Milano, Italy

R. Berlich, W. Blum, V. Büscher, H. Dietl, G. Ganis, C. Gotzhein, H. Kroha, G. Lütjens, G. Lutz, C. Mannert, W. Männer, H.-G. Moser, R. Richter, A. Rosado-Schlosser, S. Schael, R. Settles, H. Seywerd, H. Stenzel, W. Wiedenmann, G. Wolf

Max-Planck-Institut für Physik, Werner-Heisenberg-Institut, 80805 München, Fed. Rep. of Germany ${ }^{16}$

J. Boucrot, O. Callot, ${ }^{2}$ S. Chen, Y. Choi, ${ }^{21}$ A. Cordier, M. Davier, L. Duflot, J.-F. Grivaz, Ph. Heusse, A. Höcker, A. Jacholkowska, D.W. Kim, ${ }^{12}$ F. Le Diberder, J. Lefrançois, A.-M. Lutz, I. Nikolic, M.H. Schune, E. Tournefier, J.-J. Veillet, I. Videau, D. Zerwas

Laboratoire de l'Accélérateur Linéaire, Université de Paris-Sud, IN ${ }^{2} P^{3}$-CNRS, 91405 Orsay Cedex, France

P. Azzurri, G. Bagliesi, ${ }^{2}$ G. Batignani, S. Bettarini, C. Bozzi, G. Calderini, M. Carpinelli, M.A. Ciocci, V. Ciulli, R. Dell'Orso, R. Fantechi, I. Ferrante, L. Foà, ${ }^{1}$ F. Forti, A. Giassi, M.A. Giorgi, A. Gregorio, F. Ligabue, A. Lusiani, P.S. Marrocchesi, A. Messineo, F. Palla, G. Rizzo, G. Sanguinetti, A. Sciabà, J. Steinberger, R. Tenchini, G. Tonelli, ${ }^{19}$ C. Vannini, A. Venturi, P.G. Verdini

Dipartimento di Fisica dell'Università, INFN Sezione di Pisa, e Scuola Normale Superiore, 56010 Pisa, Italy

G.A. Blair, L.M. Bryant, J.T. Chambers, M.G. Green, T. Medcalf, P. Perrodo, J.A. Strong, J.H. von Wimmersperg-Toeller

Department of Physics, Royal Holloway \& Bedford New College, University of London, Surrey TW20 OEX, United Kingdom ${ }^{10}$

D.R. Botterill, R.W. Clifft, T.R. Edgecock, S. Haywood, P.R. Norton, J.C. Thompson, A.E. Wright Particle Physics Dept., Rutherford Appleton Laboratory, Chilton, Didcot, Oxon OX11 OQX, United Kingdom $^{10}$ 
B. Bloch-Devaux, P. Colas, S. Emery, W. Kozanecki, E. Lançon, M.C. Lemaire, E. Locci, P. Perez, J. Rander, J.-F. Renardy, A. Roussarie, J.-P. Schuller, J. Schwindling, A. Trabelsi, B. Vallage

CEA, DAPNIA/Service de Physique des Particules, CE-Saclay, 91191 Gif-sur-Yvette Cedex, France ${ }^{17}$

S.N. Black, J.H. Dann, R.P. Johnson, H.Y. Kim, N. Konstantinidis, A.M. Litke, M.A. McNeil, G. Taylor

Institute for Particle Physics, University of California at Santa Cruz, Santa Cruz, CA 95064, USA ${ }^{22}$

C.N. Booth, C.A.J. Brew, S. Cartwright, F. Combley, M.S. Kelly, M. Lehto, J. Reeve, L.F. Thompson

Department of Physics, University of Sheffield, Sheffield S3 7RH, United Kingdom ${ }^{10}$

K. Affholderbach, A. Böhrer, S. Brandt, G. Cowan, C. Grupen, P. Saraiva, L. Smolik, F. Stephan

Fachbereich Physik, Universität Siegen, 57068 Siegen, Fed. Rep. of Germany ${ }^{16}$

M. Apollonio, L. Bosisio, R. Della Marina, G. Giannini, B. Gobbo, G. Musolino

Dipartimento di Fisica, Università di Trieste e INFN Sezione di Trieste, 34127 Trieste, Italy

J. Rothberg, S. Wasserbaech

Experimental Elementary Particle Physics, University of Washington, WA 98195 Seattle, U.S.A.

S.R. Armstrong, E. Charles, P. Elmer, D.P.S. Ferguson, Y. Gao, S. González, T.C. Greening, O.J. Hayes, H. Hu, S. Jin, P.A. McNamara III, J.M. Nachtman, ${ }^{24}$ J. Nielsen, W. Orejudos, Y.B. Pan, Y. Saadi, I.J. Scott, J. Walsh, Sau Lan Wu, X. Wu, J.M. Yamartino, G. Zobernig

Department of Physics, University of Wisconsin, Madison, WI 53706, USA ${ }^{11}$

${ }^{1}$ Now at CERN, 1211 Geneva 23, Switzerland.

${ }^{2}$ Also at CERN, 1211 Geneva 23, Switzerland.

${ }^{3}$ Also at Dipartimento di Fisica, INFN, Sezione di Catania, Catania, Italy.

${ }^{4}$ Also Istituto di Fisica Generale, Università di Torino, Torino, Italy.

${ }^{5}$ Also Istituto di Cosmo-Geofisica del C.N.R., Torino, Italy.

${ }^{6}$ Supported by the Commission of the European Communities, contract ERBCHBICT941234.

${ }^{7}$ Supported by CICYT, Spain.

${ }^{8}$ Supported by the National Science Foundation of China.

${ }^{9}$ Supported by the Danish Natural Science Research Council.

${ }^{10}$ Supported by the UK Particle Physics and Astronomy Research Council.

${ }^{11}$ Supported by the US Department of Energy, grant DE-FG0295-ER40896.

${ }^{12}$ Permanent address: Kangnung National University, Kangnung, Korea.

${ }^{13}$ Supported by the US Department of Energy, contract DE-FG05-92ER40742.

${ }^{14}$ Supported by the US Department of Energy, contract DE-FC05-85ER250000.

${ }^{15}$ Permanent address: Universitat de Barcelona, 08208 Barcelona, Spain.

${ }^{16}$ Supported by the Bundesministerium für Bildung, Wissenschaft, Forschung und Technologie, Fed.

Rep. of Germany.

${ }^{17}$ Supported by the Direction des Sciences de la Matière, C.E.A.

${ }^{18}$ Supported by Fonds zur Förderung der wissenschaftlichen Forschung, Austria.

${ }^{19}$ Also at Istituto di Matematica e Fisica, Università di Sassari, Sassari, Italy.

${ }^{20}$ Now at Schweizerischer Bankverein, Basel, Switzerland.

${ }^{21}$ Permanent address: Sung Kyun Kwan University, Suwon, Korea.

${ }^{22}$ Supported by the US Department of Energy, grant DE-FG03-92ER40689.

${ }^{23}$ Now at School of Operations Research and Industrial Engineering, Cornell University, Ithaca, NY 14853-3801, U.S.A.

${ }^{24}$ Now at University of California at Los Angeles (UCLA), Los Angeles, CA 90024, U.S.A. 


\section{Introduction}

Neutrinos play an important role in particle physics, astrophysics and cosmology. They are a potential window towards physics beyond the Standard Model, carriers of radiated energy in star evolution, candidates for dark matter and ingredients in models of evolution of the universe.

Massive neutrinos can arise from many different mechanisms [1] but they are generally present in all extensions of the Standard Model. Among the possible frameworks the seesaw mechanism [2] is considered to be particularly appealing because it explains the smallness of neutrino masses by connecting them to the scale of new physics. See-saw models usually assume a neutrino mass hierarchy (either linear or quadratic) similar to that of up-quarks or charged leptons, thus making the tau neutrino the heaviest of the known neutrino species.

Cosmological arguments [3] limit the mass of a stable tau neutrino below a few $\mathrm{eV} / \mathrm{c}^{2}$, a region accessible only to neutrino oscillation experiments. For unstable neutrinos the cosmological bound is less stringent and depends on the nature of the decay. If the decay mode is a non-radiative one, as into neutrinos or into one neutrino and a more exotic particle like the majoron or the goldstino, the few $\mathrm{MeV} / \mathrm{c}^{2}$ mass region is no longer excluded [4]. Such a neutrino could also improve the agreement of the big-bang nucleosynthesis model with present data [4]. Decays of the type $\nu_{\tau} \rightarrow \nu_{i}+\nu_{j}+\bar{\nu}_{k}$ and $\nu_{\tau} \rightarrow \nu_{i}+j$, where $j$ is a light boson, are directly related to $\tau \rightarrow \ell_{i}+\ell_{j}+\bar{\ell}_{k}$ and $\tau \rightarrow \ell_{i}+j$, for which experimental bounds from $e^{+} e^{-}$experiments [5] exist. In this way a region of the $\left(m_{\nu_{\tau}}, \tau_{\nu_{\tau}}\right)$ plane can be excluded.

Indirect bounds on the mass of $\nu_{\tau}$ can also be derived from the decay rates of the tau. The most stringent of these limits is $m_{\nu}<48 \mathrm{MeV} / c^{2}$ at $95 \%$ confidence level (CL) [6]. Direct bounds have been derived from the reconstruction of multi-hadronic decays of the tau and several experiments [7, 8, 9] have obtained similar limits of about $30 \mathrm{MeV} / c^{2}$. The upper limit on the tau neutrino mass is currently $24 \mathrm{MeV} / c^{2}$ at $95 \% \mathrm{CL}$ and has been derived by ALEPH from $\tau^{-} \rightarrow 3 \pi^{-} 2 \pi^{+}\left(\pi^{0}\right) \nu_{\tau}{ }^{1}$ decays [10].

In this paper the $\tau^{-} \rightarrow 3 \pi^{-} 2 \pi^{+}\left(\pi^{0}\right) \nu_{\tau}$ analysis has been extended to the full data sample collected during the LEP phase I. In addition, the same technique is also applied to the decays of the tau into three prongs, $\tau^{-} \rightarrow 2 \pi^{-} \pi^{+} \nu_{\tau}$ and the two limits are combined, to give a more stringent bound on the tau neutrino mass.

Combining the above information as in reference [11] only a relatively small region of the $\left(m_{\nu}, \tau_{\nu}\right)$ plane, shown in Fig. 1, remains allowed by present data.

\section{The method}

The bound on the neutrino mass limit is derived using the method described in [10]. The tau decay is described as a two-body decay:

$$
\tau^{-}\left(E_{\tau}, \vec{p}_{\tau}\right) \rightarrow h^{-}\left(E_{h}, \overrightarrow{p_{h}}\right)+\nu_{\tau}\left(E_{\nu}, \overrightarrow{p_{\nu}}\right)
$$

\footnotetext{
${ }^{1}$ Charge conjugation is always implied throughout this paper.
} 
where the hadronic system $h^{-}$is composed of three, five or six pions. In the tau rest frame the energy of the hadronic system is fixed by the values of the masses $m_{\tau}, m_{\nu}$ and $m_{h}$ :

$$
E_{h}^{*}=\frac{m_{\tau}^{2}+m_{h}^{2}-m_{\nu}^{2}}{2 m_{\tau}}
$$

The value of $m_{\nu}$ can be computed from the above expression for given values of $m_{h}$ and $E_{h}^{*}$. In the laboratory frame the hadronic energy is

$$
E_{h}=\gamma\left(E_{h}^{*}+\beta p_{h}^{*} \cos \theta\right)
$$

where $\beta=\sqrt{\left(E_{\tau}^{2}-m_{\tau}^{2}\right) / E_{\tau}^{2}}$ is the tau velocity, $\gamma=\sqrt{1 /\left(1-\beta^{2}\right)}$ and $\theta$ is the angle between the direction of the tau and that of the hadronic system in the tau rest frame. The tau energy is assumed to be equal to the beam energy. Initial and final state radiation, which reduce the tau energy, are properly taken into account in the fit procedure, as explained later.

Since the tau direction is not determined, the neutrino mass cannot be computed directly. However the value of $E_{h}$ partially recovers the loss of information. The value of $E_{h}$ must fall inside the interval $E_{h}^{\max , \min }=\gamma\left(E_{h}^{*} \pm \beta p_{h}^{*}\right)$; this defines the kinematic allowed regions shown in Fig. 2 for different values of the tau neutrino mass. Two hypothetical events are also drawn as points with error ellipses. The ellipsoidal shape of the error is due to the measurement correlation, $\rho$, between the values of $m_{h}$ and $E_{h}$. Since both $m_{h}$ and $E_{h}$ are determined from the measured momenta of the particles composing the hadronic system a positive correlation arises between these two quantities. The value of $\rho$ is approximately $0.5-0.7$ for all the modes considered in this analysis. The size of the two ellipses in Fig. 2 is set using the average values of $\sigma_{m_{h}}, \sigma_{E_{h}}$ and $\rho$ in the $\tau^{-} \rightarrow 3 \pi^{-} 2 \pi^{+} \nu_{\tau}$ mode. Event 2 clearly constrains the neutrino mass more than event 1 , even though the latter has a higher hadronic mass. This illustrates the advantage of fitting the distribution of $E_{h}$ and $m_{h}$ rather than $m_{h}$ alone.

The density of events in the $\left(m_{h}, E_{h}\right)$ plane is essentially determined by the distribution $d \Gamma / d m_{h}$ as for fixed values of $m_{h}$ and $E_{\tau}$, the $E_{h}$ distribution is uniform between $E_{h}^{\text {min }}$ and $E_{h}^{\max }$. In the proximity of the $\mathrm{Z}$ pole the distortions caused by initial and final state radiation and by tau polarisation (which introduces a slope) are small.

The neutrino mass is extracted from a maximum likelihood fit to the analytical expression of $1 / \Gamma \cdot d^{2} \Gamma / d E_{h} d m_{h}$, after this has been convolved with radiative corrections [12] and detector effects. The expression used in the fit is:

$$
\frac{1}{\Gamma} \cdot \frac{d^{2} \Gamma}{d m_{h} d E_{h}} \propto \overline{|\mathcal{M}|^{2}}\left(m_{\tau}^{2}, m_{h}^{2}, m_{\nu}^{2}\right) \cdot \lambda^{1 / 2}\left(m_{\tau}^{2}, m_{h}^{2}, m_{\nu}^{2}\right)
$$

In the notation of Tsai [13], the matrix element $\mathcal{M}$ is:

$$
\left[p_{\alpha} k_{\beta}\left(g^{\alpha \mu} g^{\beta \nu}+g^{\alpha \nu} g^{\beta \mu}-g^{\alpha \beta} g^{\mu \nu}\right)+i k_{\alpha} p_{\beta} \varepsilon^{\alpha \mu \beta \nu}\right] \cdot\left[\left(g_{\mu \nu}-q_{\mu} q_{\nu} / q^{2}\right) \cdot v\left(q^{2}\right)+q_{\mu} q_{\nu} \cdot a\left(q^{2}\right)\right]
$$

where $p$ and $k$ are the four-momentum of the $\tau$ and of the $\nu_{\tau}$ respectively, $q^{2}=(p-k)^{2}=$ $m_{h}^{2}, v\left(q^{2}\right)$ and $a\left(q^{2}\right)$ are the spectral functions for vector and scalar final states respectively. 
This gives:

$$
\begin{gathered}
\overline{|\mathcal{M}|^{2}}=\frac{1}{2}\left[\omega_{1}\left(m_{\tau}^{2}, q^{2}, m_{\nu}^{2}\right) \cdot v\left(q^{2}\right)+\omega_{0}\left(m_{\tau}^{2}, q^{2}, m_{\nu}^{2}\right) \cdot a\left(q^{2}\right)\right] \\
\omega_{1}\left(m_{\tau}^{2}, q^{2}, m_{\nu}^{2}\right)=\left(m_{\tau}^{2}+2 q^{2}\right)\left(m_{\tau}^{2}-q^{2}\right)-m_{\nu}^{2}\left(2 m_{\tau}^{2}-q^{2}\right)+m_{\nu}^{4} \\
\omega_{0}\left(m_{\tau}^{2}, q^{2}, m_{\nu}^{2}\right)=m_{\tau}^{2}\left(m_{\tau}^{2}-q^{2}\right)-m_{\nu}^{2}\left(2 m_{\tau}^{2}+q^{2}\right)+m_{\nu}^{4}
\end{gathered}
$$

The phase space term $\lambda^{1 / 2}\left(m_{\tau}^{2}, m_{h}^{2}, m_{\nu}^{2}\right)$ is given by:

$$
\lambda^{1 / 2}\left(m_{\tau}^{2}, q^{2}, m_{\nu}^{2}\right)=\sqrt{\left(m_{\tau}^{2}-q^{2}\right)^{2}-2 m_{\nu}^{2}\left(m_{\tau}^{2}+q^{2}\right)+m_{\nu}^{4}} \simeq\left(m_{\tau}^{2}-q^{2}\right)-m_{\nu}^{2} \cdot \frac{m_{\tau}^{2}+q^{2}}{m_{\tau}^{2}-q^{2}}+\mathcal{O}\left(m_{\nu}^{4}\right)
$$

The above formulae show that the effects of $m_{\nu}$ in the matrix element are extremely small and that the sensitivity to a massive neutrino increases rapidly near $q^{2}=m_{\tau}^{2}$.

The exact functional form of the spectral functions entering the expression for $\mathcal{M}$ is not predicted by theory nor can it be inferred from $e^{+} e^{-}$data via CVC [14] as is done for modes with two or four pions in the final state. For three- and five-prong modes this is not possible because the current is axial. For the six-prong mode the CVC prescription alone is insufficient, as explained in [15]. Nevertheless, since the spectral functions are expected to vary slowly with $q^{2}$ in the small region close to the kinematic boundary, the uncertainty in their form plays only a minor role in the determination of the bound on $m_{\nu}$.

In presence of neutrino mixing the distribution of $m_{h}$ would be the incoherent superposition of three distributions:

$$
\frac{1}{\Gamma} \cdot \frac{d^{2} \Gamma}{d m_{h} d E_{h}} \propto \sum_{j}\left|V_{\tau j}\right|^{2} \cdot \overline{|\mathcal{M}|^{2}}\left(m_{\tau}^{2}, m_{h}^{2}, m_{\nu_{j}}^{2}\right) \cdot \lambda^{1 / 2}\left(m_{\tau}^{2}, m_{h}^{2}, m_{\nu_{j}}^{2}\right)
$$

where $V_{\tau j}$ is the appropriate neutrino mixing matrix element. The contributions from the two lighter neutrinos are bound by the data of oscillation experiments like E531 [16] and the experiments at the Bugey Reactor [17] to be at most of the order of $10^{-3}$ in the large $\Delta m$ region. Therefore the bound on $m_{\nu_{\tau}}$ has been determined neglecting the mixing between the tau and other families.

\section{The ALEPH detector}

The ALEPH detector and its performance are described in detail in [18, 19, 20]. A brief description of the elements of the apparatus relevant to the present analysis is given here.

Charged particles are tracked in an axial magnetic field of $1.5 \mathrm{~T}$ using a silicon microstrip vertex detector with two-dimensional readout, a drift chamber and a time projection chamber (TPC). This combined tracking system provides up to 31 coordinates and up to 338 measurements of the specific ionization for each track. For high momentum particles the transverse momentum resolution is $\Delta p_{T} / p_{T}=6 \cdot 10^{-4} p_{T}(\mathrm{GeV} / c)$. The mass resolution for a multibody decay, such as $D^{0} \rightarrow K^{-} \pi^{-} \pi^{+} \pi^{+}$, is typically of the order of $10 \mathrm{MeV} / c^{2}$. 
Surrounding the tracking detectors are the electromagnetic calorimeter (ECAL), the superconducting solenoid, the hadron calorimeter (HCAL) and the muon chambers. The ECAL is a lead wire-chamber calorimeter with cathode pad readout in $0.9^{\circ} \times 0.9^{\circ}$ projective towers divided into three longitudinal segments, with an energy resolution of $\sigma_{E} / E=0.18 / \sqrt{E(\mathrm{GeV})}+0.009$. The fine segmentation of the ECAL is relevant for photon identification and $\pi^{0}$ reconstruction. The HCAL is formed by $1.2 \mathrm{~m}$ of iron, composing the magnet return yoke, interleaved with 23 layers of streamer tubes, while the muon chambers consist of four layers of streamer tubes.

Charged particle (electron, muon, hadron) identification is performed with a likelihood method using the combined information of all subdetectors [21], while photons are reconstructed from ECAL clusters [22].

\section{Data selection}

The data selection is aimed at introducing the smallest possible bias towards lower values for the determination of the upper limit. Since at LEP the separation of $\tau^{+} \tau^{-}$events from other processes is relatively easy, the main concern is the rejection of background from misidentified tau decays. The topology of the background which lowers the neutrino mass limit is the one with a true final state multiplicity lower than the observed one as, in this case, the reconstructed values of the hadronic mass and energy are systematically higher than the true ones. The event selection has been designed to reduce such contamination to a negligible level. A moderate background from tau decays with multiplicities higher than the observed one has been tolerated whenever the loss of efficiency implied by the full background rejection was judged to be too large.

The analyses presented here are based on the data collected by ALEPH from 1991 to 1995 in the proximity of the $\mathrm{Z}$ resonance. The events were registered by means of a redundant trigger system with efficiency very close to $100 \%$. Subsequently they were filtered offline with the standard ALEPH $\tau^{+} \tau^{-}$selection [23] which retains $93.2 \%$ of the $\tau$ pairs inside the geometrical acceptance of $84.2 \%$. The contamination of this selection from $q \bar{q}$ events amounts to $0.25 \%$.

The selected events were divided into hemispheres by a plane perpendicular to the thrust axis. Each hemisphere was classified on the basis of the number of charged particles, reconstructed $\pi^{0}$ 's, and unpaired (residual) photons. Charged particles are defined as good if they have at least $100 \mathrm{MeV} / c$ momentum, at least four TPC hits, polar angle $|\cos \theta|<0.95$, and originate from a $10 \mathrm{~cm}$ long, $1 \mathrm{~cm}$ wide cylinder centred at the nominal beam interaction point. Photons are defined as good if they survive a cut on a likelihood estimator, described in [24], which takes into account the characteristics of the shower in the ECAL. Neutral pions are defined as good if they survive a cut on a likelihood estimator, also described in [24], based on the previous photon estimator and on the quality of a fit constraining the invariant mass of the two daughter photons. In contrast to the analysis in [24], events with a $\pi^{0}$ candidate in which the two daughter photons were not resolved but recognised from the ECAL cluster shape were rejected. 


\begin{tabular}{|c|c|c|c|c|}
\hline \multicolumn{2}{|c|}{} & $2 \pi^{-} \pi^{+} \nu_{\tau}$ & $3 \pi^{-} 2 \pi^{+} \nu_{\tau}$ & $3 \pi^{-} 2 \pi^{+} \pi^{0} \nu_{\tau}$ \\
\hline \hline Selection efficiency & & 49.0 & 24.7 & 7.0 \\
\hline \hline \multirow{3}{*}{$\tau$ Background } & Lower Multiplicity & 0.1 & 0.3 & 0.3 \\
& Higher Multiplicity & 6.4 & 7.1 & - \\
& Nuclear Interactions & 0.2 & 0.2 & 0.3 \\
\hline \hline$q \bar{q}$ Background & & 0.3 & 0.1 & 0.1 \\
\hline
\end{tabular}

Table 1: Selection efficiencies and contaminations (in \%). The background for $\tau^{-} \rightarrow$ $3 \pi^{-} 2 \pi^{+}\left(\pi^{0}\right) \nu_{\tau}$ refers to the whole $\left(m_{h}, E_{h}\right)$ plane while for $\tau^{-} \rightarrow 2 \pi^{-} \pi^{+} \nu_{\tau}$ is evaluated in the fitted region only.

Decays with three (five) good charged tracks, no other charged tracks, no good $\pi^{0}$ and no good residual photons were classified as $\tau^{-} \rightarrow 2 \pi^{-} \pi^{+} \nu_{\tau}\left(\tau^{-} \rightarrow 3 \pi^{-} 2 \pi^{+} \nu_{\tau}\right)$ candidates. Decays with five good charged tracks, no other charged tracks, one good $\pi^{0}$ and no residual photons were classified as $\tau^{-} \rightarrow 3 \pi^{-} 2 \pi^{+} \pi^{0} \nu_{\tau}$. Decays with one or more identified electrons or with a pair of tracks compatible with a photon converting inside the tracking volume were rejected. From the four-vectors of the charged tracks and of the reconstructed $\pi^{0}$ the invariant mass, $m_{h}$, and the total energy, $E_{h}$, were computed assuming that all particles are pions, so that tau decays involving kaons are reconstructed with slightly smaller invariant mass and energy. No attempt to identify kaons is made.

Additional cuts are applied to increase the purity of the selected sample. The sum of the absolute values of the impact parameters of the charged tracks is required to be less than $0.8 \mathrm{~cm}$ in the five-prong mode and less than $0.6 \mathrm{~cm}$ in the three-prong mode; the recoiling hemisphere is required to have fewer than four charged tracks and a total invariant mass, built from both charged particles and photon candidates, smaller than the tau mass. Finally the total electric charge of the event is required to be null or \pm 1 .

Due to the large number of candidates the selection and the fit in the $\tau^{-} \rightarrow 2 \pi^{-} \pi^{+} \nu_{\tau}$ channel are restricted to the region of the $\left(E_{h}, m_{h}\right)$ plane $0.89<E_{h} / E_{\text {beam }}<1.07$ and $0.76<m_{h}<1.83 \mathrm{GeV} / c^{2}$. The fitted region is shown in Fig. 3. The size of region has been chosen large enough to make the limit on the tau neutrino mass insensitive to variation of the region boundaries. All the figures concerning the $\tau^{-} \rightarrow 2 \pi^{-} \pi^{+} \nu_{\tau}$ channel given in the following refer the fitted region only.

The efficiency and the contamination for the $\tau^{-} \rightarrow 2 \pi^{-} \pi^{+} \nu_{\tau}, \tau^{-} \rightarrow 3 \pi^{-} 2 \pi^{+} \nu_{\tau}$ and $\tau^{-} \rightarrow 3 \pi^{-} 2 \pi^{+} \pi^{0} \nu_{\tau}$ channels are reported in Table 1 . The lower efficiency of the last mode is caused by stringent cuts on $\pi^{0}$ reconstruction which are needed to suppress the cross-channel contamination from $\tau^{-} \rightarrow 3 \pi^{-} 2 \pi^{+} \nu_{\tau}$. The background from tau decays has been divided into three categories: events from modes with true final state multiplicity higher than that of the signal, modes with lower multiplicity and events where the multiplicity is modified because one or more charged particles interacted with the material of the detector. The efficiencies and the backgrounds have been estimated by reconstructing events generated with the KORALZ [25] program with a GEANT [26] 
based simulation of the ALEPH detector. The variation of the efficiencies in the $\left(E_{h}, m_{h}\right)$ plane is discussed in section 6.3.

A total of $2939 \tau^{-} \rightarrow 2 \pi^{-} \pi^{+} \nu_{\tau}$ candidates and $52(3) \tau^{-} \rightarrow 3 \pi^{-} 2 \pi^{+}\left(\pi^{0}\right) \nu_{\tau}$ candidates are selected in the data in good agreement with the expectations of 2908 $\tau^{-} \rightarrow 2 \pi^{-} \pi^{+} \nu_{\tau}$ and 50 (4) $\tau^{-} \rightarrow 3 \pi^{-} 2 \pi^{+}\left(\pi^{0}\right) \nu_{\tau}$ decays based on the PDG [5] average branching fractions. The distributions in the upper part of the $\left(E_{h}, m_{h}\right)$ plane are shown in Fig. 3 and Fig. 4.

\section{The Likelihood Function}

The likelihood equations for the $\tau^{-} \rightarrow 3 \pi^{-} 2 \pi^{+}\left(\pi^{0}\right) \nu_{\tau}$ and the $\tau^{-} \rightarrow 2 \pi^{-} \pi^{+} \nu_{\tau}$ modes have been treated differently. In the first case an unbinned likelihood fit was performed while in the second, due to the large number of events, the $\left(E_{h}, m_{h}\right)$ plane has been divided into bins. The size of the bins has been chosen similar to the energy and mass resolutions in most of the $\left(m_{h}, E_{h}\right)$ plane, and decreased in the region of the plane more sensitive to a massive neutrino. Several other binnings have been used to check that the fit does not depend on a particular choice. In each bin the Poisson probability to find the observed number of events has been calculated. In both fits the probability density is:

$$
\begin{gathered}
\mathcal{P}\left(m_{\nu}\right)=\frac{1}{\Gamma} \cdot \frac{d^{2} \Gamma}{d E_{h} d m_{h}} \otimes \mathcal{G}\left(E_{\text {beam }}, E_{\tau}\right) \otimes \mathcal{R}\left(m_{h}, E_{h}, \rho, \sigma_{m_{h}}, \sigma_{E_{h}}, \ldots\right) \otimes \varepsilon\left(m_{h}, E_{h}\right) \\
\mathcal{L}=\prod_{i}^{N} \mathcal{P}_{i}\left(m_{\nu}\right)
\end{gathered}
$$

where $\mathcal{G}\left(E_{\text {beam }}, E_{\tau}\right)$ is the radiation kernel, $\mathcal{R}\left(m_{h}, E_{h}, \rho, \sigma_{m_{h}}, \sigma_{E_{h}}, \ldots\right)$ and $\varepsilon\left(m_{h}, E_{h}\right)$ are the detector resolution and the selection efficiency of each mode respectively. The expressions used for $\mathcal{R}$ are described in section 6.2. Events outside the kinematic boundary contribute to the likelihood only through the detector resolution or the initial/final state radiation kernel; hence the upper limit derived from the fit is not sensitive to events with hadronic energies or masses which are many standard deviations away from the kinematic boundary.

The fits to the $2939 \tau^{-} \rightarrow 2 \pi^{-} \pi^{+} \nu_{\tau}$ and to the $55 \tau^{-} \rightarrow 3 \pi^{-} 2 \pi^{+}\left(\pi^{0}\right) \nu_{\tau}$ events give $95 \% \mathrm{CL}$ upper limits on the tau neutrino mass of $22.3 \mathrm{MeV} / c^{2}$ and $21.5 \mathrm{MeV} / c^{2}$ respectively. The two likelihoods are shown in Fig. 5 and Fig. 6 . The $95 \%$ confidence level is taken as the point where the logarithm of the likelihood is 1.92 lower than its maximum.

A possible bias in the fit procedure was investigated by fitting Monte Carlo samples with massive neutrinos. In all cases the fits were correctly sensitive to the input neutrino mass. For the $\tau^{-} \rightarrow 2 \pi^{-} \pi^{+} \nu_{\tau}$ channel the likelihood distributions of three samples with 0,20 and $40 \mathrm{MeV} / c^{2}$ input neutrino mass were fitted finding preferred values of the neutrino mass and $95 \% \mathrm{CL}$ intervals of $2.4_{-2.4}^{+10.6} \mathrm{MeV} / c^{2}, 17.7_{-17.7}^{+20.0} \mathrm{MeV} / c^{2}$ and $44.5_{-23.6}^{+11.6} \mathrm{MeV} / c^{2}$ respectively. The samples correspond to about 3.5, 1 and 1 times the 
data statistics; the minus log-likelihood distributions for the last two samples are shown in Fig. 7. For the $\tau^{-} \rightarrow 3 \pi^{-} 2 \pi^{+} \nu_{\tau}$ channel, two samples corresponding to three times the data statistics, with input masses of 30 and $60 \mathrm{MeV} / c^{2}$ were fitted giving neutrino masses of $34.2_{-34.2}^{+23.5} \mathrm{MeV} / c^{2}$ and $69.1_{-13.6}^{+19.6} \mathrm{MeV} / c^{2}$ respectively (again the errors refer to $95 \%$ CL intervals).

\section{Systematic Uncertainties}

Several sources of systematic errors have been considered. For each source a new fit was performed, having changed in the likelihood the appropriate quantity by one standard deviation. The difference between the value of the $95 \% \mathrm{CL}$ upper limit on $m_{\nu_{\tau}}$ obtained from the original fit and the one with the modified likelihood has been taken as the systematic error due to that source. All the variations were then summed in quadrature to give the global systematic error which was added linearly to the result of the original fit. In principle the unmodified likelihood had to be convolved with the error probability density function of each source of error [27]. Since the number of error sources is very large and affects the likelihood through complicated expressions, the standard procedure is numerically clumsy. However, the procedure used in this work is conservative, in the sense that each individual one-sigma shift produces a variation of the upper limit on the tau neutrino mass larger than the one obtained convolving the likelihood expression with an additional gaussian error probability density function.

The sources of systematics considered belong to four major categories: tau properties, such as tau mass, energy and polarisation; detector effects, such as absolute momentum calibration and resolution; selection efficiency and background contamination; and tau decay modelling. The corresponding variations of the neutrino mass limit are reported in Table 2 .

\subsection{Tau properties}

The values of the tau mass and polarisation have been varied according to the uncertainties quoted in [36] and [37] respectively. The tau energy is assumed to be equal to the beam energy on which the error given in [38] was assumed. The impact of these systematics on the tau neutrino mass limit is small.

\subsection{Detector effects}

Detector effects concern mainly two aspects: the parametrisation of the resolution function $\mathcal{R}$ and the calibration of the detector.

The form of $\mathcal{R}$ has been determined differently for the $\tau^{-} \rightarrow 3 \pi^{-} 2 \pi^{+}\left(\pi^{0}\right) \nu_{\tau}$ and the $\tau^{-} \rightarrow 2 \pi^{-} \pi^{+} \nu_{\tau}$ decays. In the first case each event defined a precise kinematic configuration which was used as input to generate many thousands of identical Monte Carlo events. These events were then passed through the full detector simulation and 
reconstructed. This procedure predicts a Gaussian shape for $\mathcal{R}$, with a resolution in $m_{h}$ about 1.4 times larger than that computed directly from the tracking error matrix, a consequence of the very special topology of these events. Since the value of the invariant mass and energy are correlated, the function used to describe $\mathcal{R}$ is a two-dimensional Gaussian depending on three parameters $\left(\sigma_{m}, \sigma_{E}\right.$ and $\left.\rho\right)$. Small non-Gaussian tails were also found and have been taken into account in the expression of $\mathcal{R}$ up to $\pm 10 \sigma_{m, E}$. These tails originate from hard scattering in the subdetector walls, incorrect hit assignments in the vertex detector and in the inner drift chamber.

In the case of $\tau^{-} \rightarrow 2 \pi^{-} \pi^{+} \nu_{\tau}$ there are too many candidates to apply the same procedure. Therefore the expression for $\mathcal{R}$ was derived as a function of $m_{h}$ and $E_{h}$ in each bin of the $\left(m_{h}, E_{h}\right)$ plane. Since $\mathcal{R}$ is a function of the pion four-momenta $\vec{p}_{i}$ this procedure averages different kinematic configurations giving the same values of $m_{h}$ and $E_{h}$. However $\mathcal{R}$ is well described by the sum of two (two-dimensional) Gaussians with the addition of a $7 \sigma$ flat tail. The dependence on $m_{h}$ and $E_{h}$ of each parameter entering the expression for $\mathcal{R}$ has been studied and taken into account. For both modes different parametrisations of $\mathcal{R}$ have been tried by varying the extent and the shape of the non-Gaussian tails. For completeness, several events lying near the kinematic boundary have been specially investigated by using the duplication technique used for the $\tau^{-} \rightarrow 3 \pi^{-} 2 \pi^{+}\left(\pi^{0}\right) \nu_{\tau}$ events.

The mean values and the resolutions entering the expressions for $\mathcal{R}$ have been varied to take into account possible calibration errors. For the final states with only charged pions the correct momentum calibration is important as both the total energy and the invariant mass are computed from the measured momenta. The calibration of the absolute value of the momentum uses a parametrization of the deviations from the measured values of the form:

$$
\Delta=\left|\vec{p}-\vec{p}_{0}\right|=k_{1} \cdot|\vec{p}|+q \cdot k_{2} \cdot|\vec{p}|^{2}
$$

where $\vec{p}$ is the measured momentum, $\vec{p}_{0}$ the true momentum, $q$ the electric charge and $k_{1}$ and $k_{2}$ two constants related to distortions in the magnetic field and to sagitta errors respectively. The two effects have been disentangled by measuring quantities proportional to the sum or the difference of momenta of oppositely charged particles with similar momenta.

The value of $k_{1}=(2 \pm 3) \times 10^{-4}$ was derived from the measurement of the $D^{0}$ mass in $D^{0} \rightarrow K^{-} \pi^{+}$decays. For these decays, the error on the invariant mass of the kaon-pion system is dominated by the error on $\left|\vec{p}_{K}\right| \cdot\left|\vec{p}_{\pi}\right|$, which in turn is dominated by the $k_{1}$ term. The measured mass of $D^{0}$ candidates is shown in Fig. 8. The spectrum is fitted with a Gaussian for signal events and a polynomial for the background, as explained in [35]. The measured value is compatible with the current world average [5], with a statistical error smaller than $0.5 \mathrm{MeV} / c^{2}$. The upper limit for $k_{1}$ was used in computing the systematics. The value of $k_{2}=(4 \pm 5) \times 10^{-6} \mathrm{GeV} / c^{-1}$ was derived from $e^{+} e^{-} \rightarrow \mu^{+} \mu^{-}$events from the difference of muon momenta. Also in this case no net effect was observed. For typical momenta of $10-15 \mathrm{GeV} / c$, the possible effects on $m_{h}$ and $E_{h}$ due to the $k_{2}$ term are much smaller than those related to $k_{1}$.

The momentum resolution was also determined from $D^{0} \rightarrow K^{-} \pi^{+}$decays by comparing the width of the $D^{0}$ peak in the data, $\sigma_{D^{0}}^{D T}$, with the one in the Monte Carlo 
simulation, $\sigma_{D^{0}}^{M C}$, which agreed at the $10 \%$ level. A possible dependence of $k_{1}$ and of $\sigma_{D^{0}}^{D T} / \sigma_{D^{0}}^{M C}$ on the momentum of the $D^{0}$, the angle between the pion and the kaon, the polar angle of the $D^{0}$ and the data taking period were investigated. No sizeable effect was observed.

The mass of the hadronic system is also sensitive to possible miscalibration of the angular separation between the charged tracks. This effect is much less important in $D^{0} \rightarrow K^{-} \pi^{+}$decays since the opening angle between the kaon and the pion is large with respect to the angular resolution of the tracking system. In order to determine the $m_{h}$ calibration and resolution, the following decays have been used: $D^{0} \rightarrow K^{-} \pi^{+} \pi^{-} \pi^{+}$(for the $\tau^{-} \rightarrow 3 \pi^{-} 2 \pi^{+} \nu_{\tau}$ mode) and $D^{+} \rightarrow K^{-} \pi^{+} \pi^{+}$(for the $\tau^{-} \rightarrow 2 \pi^{-} \pi^{+} \nu_{\tau}$ mode). In these decays the topology of the hadronic final state is very similar to that of the corresponding tau mode. The correlation between $E_{h}$ and $m_{h}$ has been taken into account in the computation of the systematics from the above effects. The ECAL calibration for $\pi^{0}$ in $\tau^{-} \rightarrow 3 \pi^{-} 2 \pi^{+} \pi^{0} \nu_{\tau}$ events was deduced from the value of the $\rho(770)$ mass measured in $\tau^{-} \rightarrow \rho^{-} \nu_{\tau}$ decays.

\subsection{Selection efficiency and background simulation}

The selection criteria for both modes imply very loose kinematic requirements, so that the selection efficiencies are expected to be independent of $m_{h}$ and $E_{h}$. The efficiencies were mapped in the plane $\left(m_{h}, E_{h}\right)$ using simulated data. In the case of $\tau^{-} \rightarrow 2 \pi^{-} \pi^{+} \nu_{\tau}$ the efficiency depends linearly on $m_{h}$ alone. This effect arises because at larger values of $m_{h}$ the mean opening angle between the daughter tracks is larger and hence two-track confusion is reduced. In the $\tau^{-} \rightarrow 3 \pi^{-} 2 \pi^{+}\left(\pi^{0}\right) \nu_{\tau}$ mode the daughter tracks are more separated than in the $\tau^{-} \rightarrow 2 \pi^{-} \pi^{+} \nu_{\tau}$ mode and no dependence on either mass or energy is observed. The systematic error arising from the dependence of the selection efficiency upon $m_{h}$ and $E_{h}$ has been evaluated by conservatively varying the efficiency slopes by $\pm 10 \%$. The effect of the size of the fitted box region has been investigated by varying the boundaries by $20 \mathrm{MeV} / c^{2}$ in mass and by $1 \mathrm{GeV}$ in energy. The corresponding variations of the upper limit on the neutrino mass are small.

The background from non- $\tau$ events has been investigated on data by inverting some of the hemisphere selection cuts. For example, non- $\tau$ hemispheres in the five- or three-prong topology are selected requiring in the recoiling hemisphere the invariant mass to be greater than the tau mass and the number of tracks to be greater than five. Similarly five- and three-prong selected hemispheres with masses much greater than the tau mass provide an (almost) independent tag for non- $\tau$ recoiling hemispheres, so that the multiplicity and the invariant mass distribution of the background are measured. The number of non- $\tau$ background events is computed combining this information under the assumption that correlations between the hemispheres are negligible. The estimated background is found in good agreement with the prediction from fully simulated hadronic and four-fermion events and has a negligible effect on the determination of the neutrino mass limit.

In the $\tau^{-} \rightarrow 2 \pi^{-} \pi^{+} \nu_{\tau}$ analysis all sources of background from other tau decays which could mimic a massless neutrino have been reduced to a negligible level. Therefore 
the knowledge of the absolute normalisation and the shape of this background has no effect on the determination of $m_{\nu_{\tau}}$. The uncertainty on the other sources of background (mainly $\tau^{-} \rightarrow \pi^{-} \pi^{+} \pi^{-} \pi^{0} \nu_{\tau}$ and $\tau^{-} \rightarrow K^{-} \pi^{+} \pi^{-} \nu_{\tau}$ ) has been introduced by varying the global contribution by $\pm 10 \%$. In the $\tau^{-} \rightarrow 3 \pi^{-} 2 \pi^{+}\left(\pi^{0}\right) \nu_{\tau}$ analysis the probability that a $\tau^{-} \rightarrow 3 \pi^{-} 2 \pi^{+} \pi^{0} \nu_{\tau}$ candidate is in fact a $\tau^{-} \rightarrow 3 \pi^{-} 2 \pi^{+} \nu_{\tau}$ event is $7 \%$. This has been taken into account by introducing the $\tau^{-} \rightarrow 3 \pi^{-} 2 \pi^{+} \pi^{0} \nu_{\tau}$ candidates as $\tau^{-} \rightarrow 3 \pi^{-} 2 \pi^{+} \nu_{\tau}$ in the likelihood equations with a $7 \%$ statistical weight. The other sources of background are negligible.

\subsection{Spectral Functions}

The decay $\tau^{-} \rightarrow 2 \pi^{-} \pi^{+} \nu_{\tau}$ is described using the model of Kühn and Santamaria [28], inspired by the asymptotic limit $\left(q^{2} \rightarrow 0\right)$ of chiral theory. This model is implemented in the KORALZ [25] program. In its framework the scalar term entering the expression of $\mathcal{M}$ is neglected, since it is suppressed by PCAC. The vector term is fully dominated by the $a_{1}(1260)$ resonance, which decays according to the chain $a_{1}^{-} \rightarrow \rho^{0}\left(\rho^{\prime 0}\right) \pi^{-} \rightarrow \pi^{+} \pi^{-} \pi^{-}$.

The hadronic current $J_{\text {had }}^{\mu}$ is written:

$$
J_{\text {had }}^{\mu}=<\pi\left(q_{1}\right) \pi\left(q_{2}\right) \pi\left(q_{3}\right)\left|J_{\text {had }}^{\mu}(0)\right| 0>=-i \frac{2 \sqrt{2}}{3 f_{\pi}} B W_{a_{1}}\left(q^{2}\right)\left[B_{\rho}\left(s_{1}\right) \cdot V_{1}^{\mu}+B_{\rho}\left(s_{2}\right) \cdot V_{2}^{\mu}\right]
$$

where $f_{\pi}$ is the pion decay constant, $q_{j}(j=1,2)$ are the four-momenta of the two like-sign pions, $q_{3}$ is the four-momentum of the unlike-sign pion, $s_{j}=\left(q_{j}+q_{3}\right)^{2}, V_{j}^{\mu}$ and $B_{\rho}$ are given by:

$$
V_{j}^{\mu}=q_{j}^{\mu}-q_{3}^{\mu}-q^{\mu \frac{q \cdot\left(q_{j}-q_{3}\right)}{q^{2}}} \quad B_{\rho}\left(s_{j}\right)=\frac{B W_{\rho(770)\left(S_{j}\right)}+\beta B W_{\rho(1450)}\left(s_{j}\right)}{1+\beta}
$$

and $B W_{x}\left(s_{j}\right)$ are the Breit-Wigner functions with energy-dependent widths. The values of $m_{a_{1}}, \Gamma_{a_{1}}$ and $\beta$ are fitted to the ARGUS data [28].

This approach has been refined by several authors who consider the distortions to the $a_{1}$ propagator due to the effect of the $K^{*}(892) \bar{K}$ threshold [29], by introducing a pseudoscalar $\pi(1300)$ resonance [30] or a non-resonant term [31] in the decay amplitude. The inclusion of a $\pi(1300)$ term considered in [30] would introduce an additional contribution to the total width $\Gamma_{3 \pi}$ of about $5 \%$. The scalar part of the spectral function peaks at $q^{2} \simeq m_{\pi(1300)}^{2}$, becoming almost constant after $q^{2} \simeq 2.2\left(\mathrm{GeV} / c^{2}\right)^{2}$; its contribution has been recently bound by OPAL [32] to be smaller than $0.84 \%$ at $95 \%$ CL. All these improvements have a minor effect on the $\tau^{-} \rightarrow 2 \pi^{-} \pi^{+} \nu_{\tau}$ fit because they distort the invariant mass distribution slightly. Only the presence of a narrow resonance close to the mass end-point would have a significant effect on the $m_{\nu_{\tau}}$ limit determination. However, the impact of this distortion in the likelihood fit is reduced by the fact that the sensitivity to the tau neutrino mass derives mainly from the distribution of the energy rather than that of the mass. The systematic error due to the use of the Kühn-Santamaria model has been evaluated by varying, in a correlated way, the model parameters in the following ranges: $\delta m_{a_{1}}= \pm 20 \mathrm{MeV} / c^{2}, \delta \Gamma_{a_{1}}= \pm 60 \mathrm{MeV} / c^{2}$, 
$\delta m_{\rho}= \pm 5 \mathrm{MeV} / c^{2}, \delta \Gamma_{\rho}= \pm 10 \mathrm{MeV} / c^{2}, \delta m_{\rho^{\prime}}= \pm 100 \mathrm{MeV} / c^{2}$ and $\delta \Gamma_{\rho^{\prime}}= \pm 100 \mathrm{MeV} / c^{2}$. The $\tau^{-} \rightarrow 2 \pi^{-} \pi^{+} \nu_{\tau}$ data mass spectrum is shown in Fig. 9 together with the KühnSantamaria model prediction for a massless neutrino.

The situation for the $\tau^{-} \rightarrow 3 \pi^{-} 2 \pi^{+}\left(\pi^{0}\right) \nu_{\tau}$ modes is different. There are very few studies of the spectral functions, mainly because the number of observed candidates is extremely small. Experimentally it is seen that the invariant mass of the hadronic system peaks at high values of $q^{2}$ and seems unlikely to be dominated by a single resonance. In the published works by ARGUS, CLEO and OPAL [7, 8, 33] a crude model with no scalar term and pure phase space, i.e. uniform spectral functions, was used. The description can be improved by the addition of a spin-one wave. Some studies along these lines have been performed by assuming a $\tau^{-} \rightarrow \rho \rho \pi^{-} \nu_{\tau}$ decay as in [34], or either a $\tau^{-} \rightarrow a_{1}^{-} \pi^{+} \pi^{-} \nu_{\tau}$ or a $\tau^{-} \rightarrow \rho^{0} \pi^{+} \pi^{-} \pi^{-} \nu_{\tau}$ decay as in the previous ALEPH analysis [10]. In all cases the inclusion of the intermediate resonances has the effect of shifting the shape of the spectral function to higher $q^{2}$. In Fig. 10 the invariant mass distribution of the five-prong candidates is compared with those predicted by a pure phase space model and by a model with an intermediate $a_{1} \pi \pi$ state, which seems to be preferred. The numerical effects on the ALEPH bound induced by the use of either model were found to be negligible [10].

\subsection{Total Systematic Errors}

For each source of systematic error the corresponding variation of the neutrino mass limit is reported in Table 2. The major effects result from energy and mass calibration, resolution and from the modelling of the resolution functions. The dominant sources of systematics for the $\tau^{-} \rightarrow 2 \pi^{-} \pi^{+} \nu_{\tau}$ mode are the energy calibration and resolution. The sensitivity to energy miscalibration in this channel is much larger than for the $\tau^{-} \rightarrow 3 \pi^{-} 2 \pi^{+}\left(\pi^{0}\right) \nu_{\tau}$ one because the quadratic dependence on $m_{\nu_{\tau}}$ of the energy endpoint is much more important at lower $q^{2}$.

The variations for both three- and five-prong final states are separately summed in quadrature to obtain the two total systematic errors of 4.2 and $0.8 \mathrm{MeV} / c^{2}$ respectively. These errors are summed linearly to the measured mass limits to obtain 95\% CL upper limits of $25.7 \mathrm{MeV} / c^{2}$ and $23.1 \mathrm{MeV} / c^{2}$ for the three-prong and five-prong modes respectively. Interestingly the $\tau^{-} \rightarrow 2 \pi^{-} \pi^{+} \nu_{\tau}$ mode is competitive with the $\tau^{-} \rightarrow$ $3 \pi^{-} 2 \pi^{+}\left(\pi^{0}\right) \nu_{\tau}$ mode thanks to the larger number of candidates which compensate for the less favourable distribution in the $\left(E_{h}, m_{h}\right)$ plane. The two limits are complementary since the limit derived from the $\tau^{-} \rightarrow 2 \pi^{-} \pi^{+} \nu_{\tau}$ mode is more sensitive to the energy distribution and the others to the mass distribution of the hadronic system.

\section{Combined Results}

The combined upper limit has been determined from a new likelihood $\mathcal{L}^{\text {comb }}$, constructed as the product of the individual $\tau^{-} \rightarrow 2 \pi^{-} \pi^{+} \nu_{\tau}$ and $\tau^{-} \rightarrow 3 \pi^{-} 2 \pi^{+}\left(\pi^{0}\right) \nu_{\tau}$ likelihoods $\mathcal{L}^{3 \pi}$ and $\mathcal{L}^{5(6) \pi}$. This likelihood limits $m_{\nu_{\tau}}$ below $16.6 \mathrm{MeV} / c^{2}$ at $95 \% \mathrm{CL}$. 


\begin{tabular}{|c|c|c|c|}
\hline \multirow[t]{2}{*}{ Source } & \multicolumn{3}{|c|}{$\begin{array}{c}\text { Variation of } m_{\nu} \text { limit } \\
\left(\mathrm{MeV} / c^{2}\right)\end{array}$} \\
\hline & $\tau^{-} \rightarrow 3 \pi^{-} 2 \pi^{+}\left(\pi^{0}\right) \nu_{\tau}$ & 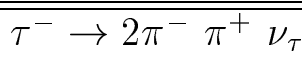 & $\overline{\text { combined }}$ \\
\hline$\tau$ mass & 0.2 & 0.3 & 0.2 \\
\hline beam energy & $<0.1$ & 0.1 & 0.2 \\
\hline$\tau$ polarisation & $<0.1$ & 0.1 & 0.1 \\
\hline slope of selection efficiency & $<0.1$ & 0.1 & 0.1 \\
\hline$\tau$ background & 0.3 & 0.1 & 0.2 \\
\hline energy-mass calibration & 0.3 & 2.6 & 0.9 \\
\hline energy-mass resolution & 0.2 & 3.1 & 1.1 \\
\hline spectral function & $<0.1$ & 0.3 & 0.1 \\
\hline modelling of resolution & 0.6 & 1.1 & 0.6 \\
\hline total & 0.8 & 4.2 & 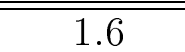 \\
\hline
\end{tabular}

Table 2: Systematic variation of the 95\% CL upper limit on $m_{\nu}$ (in $\mathrm{MeV} / c^{2}$ ) for the individual and combined $\tau^{-} \rightarrow 3 \pi^{-} 2 \pi^{+}\left(\pi^{0}\right) \nu_{\tau}$ and $\tau^{-} \rightarrow 2 \pi^{-} \pi^{+} \nu_{\tau}$ likelihoods.

The systematics on the combined upper limit were determined using $\mathcal{L}^{3 \pi}, \mathcal{L}^{5(6) \pi}$ and the two sets of modified likelihoods $\mathcal{L}_{\sigma_{i}}^{3 \pi}, \mathcal{L}_{\sigma_{i}}^{5(6) \pi}$ used for the systematics on the individual upper limits. For each source of error $i$ a modified combined likelihood $\mathcal{L}_{\sigma_{i}}^{\text {comb }}$ was derived by multiplying the two corresponding likelihoods, $\mathcal{L}_{\sigma_{i}}^{3 \pi}$ and $\mathcal{L}_{\sigma_{i}}^{5(6) \pi}$, and a new $95 \% \mathrm{CL}$ upper limit computed. The difference between this limit and the one computed with $\mathcal{L}^{\text {comb }}$ was taken as the systematic error deriving from the $i-t h$ source. When the error source affected only one of the two modes the likelihood of the other mode used in constructing $\mathcal{L}_{\sigma_{i}}^{c o m b}$ was the unmodified one. For example in the case of the three prong spectral function, the combined modified likelihood is $\mathcal{L}_{\sigma_{i}}^{\text {comb }}=\mathcal{L}_{\sigma_{i}}^{3 \pi} \cdot \mathcal{L}^{5(6) \pi}$. Table 2 summarises the variation of the two limits and the variation of the combined limit, for each source of error. In this way a total systematic error of $1.6 \mathrm{MeV} / c^{2}$ and a final $95 \%$ CL limit of $18.2 \mathrm{MeV} / c^{2}$ were obtained.

Recently the DELPHI Collaboration has suggested the existence of a hitherto unseen decay mode of the tau in a radial excitation of the $a_{1}$ [39]. In that analysis this $a^{\prime}$ resonance is assigned a mass of $1700 \mathrm{MeV} / c^{2}$ and a width of $300 \mathrm{MeV}$. Its contribution is fitted to be $(2.3 \pm 0.6) \%$. If a $2.5 \%$ of this resonance is introduced in the fit of the $\tau^{-} \rightarrow 2 \pi^{-} \pi^{+} \nu_{\tau}$ mode the agreement between the model and the data deteriorates giving a $\chi^{2} /$ n.d.f. of $1077 / 999$ with respect to the value of $1059 / 999$ obtained with the KS spectrum alone. If this resonance were considered in the fit the limit from the $\tau^{-} \rightarrow 2 \pi^{-} \pi^{+} \nu_{\tau}$ sample would increase by $6 \mathrm{MeV} / c^{2}$ and the combined limit would increase from 18.2 to $19.2 \mathrm{MeV} / c^{2}$. 


\section{Conclusions}

ALEPH has used the modes $\tau^{-} \rightarrow 2 \pi^{-} \pi^{+} \nu_{\tau}$ and $\tau^{-} \rightarrow 3 \pi^{-} 2 \pi^{+}\left(\pi^{0}\right) \nu_{\tau}$ to bound the tau neutrino mass by fitting the distribution of events in the $\left(m_{h}, E_{h}\right)$ plane. An upper limit of $18.2 \mathrm{MeV} / \mathrm{c}^{2}$ on the tau neutrino mass is obtained at $95 \%$ confidence level. This result is more stringent than previous determinations but it is not sufficient to close the window for a massive tau neutrino shown in Fig. 1.

\section{Acknowledgements}

We gratefully acknowledge our colleagues from the CERN accelerator divisions for the successful operation of LEP. We are indebted to the engineers and technicians from all our institutions for their contribution to the excellent performance of ALEPH. Those of us from non-member countries thank CERN for its hospitality. 


\section{References}

[1] G. Gelmini, E. Roulet, Rept. Prog. Phys. 58 (1995) 1207.

[2] M. Gell-Mann, P. Ramond and R. Slansky, Supergravity, Ed. by D. Freedmann et al, North Holland (1979).

[3] R. Coswik and J. McClelland, Phys. Rev. Lett. 29 (1972) 669.

[4] M. Kawasaki et. al., Nucl. Phys. B 149 (1994) 105.

[5] R. M. Barnett et al., Phys. Rev. D 54 (1996) 1.

[6] A. Bottino et al., Phys. Rev. D 53 (1996) 6361. J. Swain et al., Proceedings of the Fourth Workshop on Tau Lepton Physics, Ed. by J. G. Smith and W. Toki, North Holland (1997).

[7] ARGUS Collaboration, Phys. Lett. B 292 (1992) 221.

[8] CLEO Collaboration, Phys. Rev. D 70 (1993) 3700.

[9] OPAL Collaboration, Zeit. für Phys. C 72 (1996) 231.

[10] ALEPH Collaboration, Phys. Lett. B 349 (1995) 585.

[11] H. Harari, Proceedings of the Third Workshop on Tau Lepton Physics, Ed. by L. Rolandi, North Holland (1994).

[12] CERN 89-08, Z Physics at LEP 1, Vol. 1, Edited by G. Altarelli, R. Kleiss and C. Verzegnassi.

[13] Y. S. Tsai, Phys. Rev. D 4 (1971) 2821; Phys. Rev. D 13 (1976) 771.

[14] A. Pais, Ann. Phys. 9 (1960) 548.

[15] A. Rouge, Zeit. für Phys. C 70 (1996) 65.

[16] N. Ushida et al., Phys. Rev. Lett. B 57 (1986) 2897.

[17] B. Achkar et al., Nucl. Phys. B 434 (1995) 503.

[18] ALEPH Collaboration, N.I.M. A 294 (1990) 121; N.I.M. A 303 (1991) 393.

[19] B. Mours et al., N.I.M. A379 (1996) 101.

[20] ALEPH Collaboration, N.I.M. A 360 (1995) 481.

[21] ALEPH Collaboration, Zeit. für Phys. C 70 (1996) 561.

[22] ALEPH Collaboration, Zeit. für Phys. C 69 (1996) 183. 
[23] ALEPH Collaboration, Zeit. für Phys. C 62 (1994) 539.

[24] ALEPH Collaboration, Zeit. für Phys. C 70 (1996) 579.

[25] S. Jadach et al., Comp. Phys. Comm. 79 (1994) 503.

[26] R. Brun et al., CERN DD/EE/84-1.

[27] R. D. Cousins and V. L. Highland, N.I.M. A320 (1992) 321.

[28] J. H. Kühn et al, Zeit. für Phys. C 48 (1990) 445.

[29] N. A. Törnqvist, Zeit. für Phys. C 36 (1987) 695.

[30] J. H. Kühn et al., Zeit. für Phys. C 56 (1992) 661.

[31] N. Isgur et al., Phys. Rev. D 39 (1989) 1357.

[32] OPAL Collaboration, CERN-PPE/97-020, (1997).

[33] OPAL Collaboration, Zeit. für Phys. C 65 (1995) 183.

[34] J. J. Gomez-Cadenas et al, Phys. Rev. D 41 (1990) 2179.

[35] ALEPH Collaboration, Phys. Lett. B 388 (1996) 648.

[36] BES Collaboration, Phys. Rev. D 53 (1996) 20-34.

[37] J. C. Brient, Proceedings of Fourth Workshop on Tau Lepton Physiscs, Ed. by J. G. Smith and W. Toki, North Holland (1997).

[38] Working Group on LEP Energy, Zeit. für Phys. C 66 (1995) 567;

Phys. Lett. B 284 (1992) 431.

[39] DELPHI Collaboration, contributed papers 319(PA 7 PL 6) and 320(PA 7, 10 PL 6, 11) to HEP'97 Jerusalem 1997. 


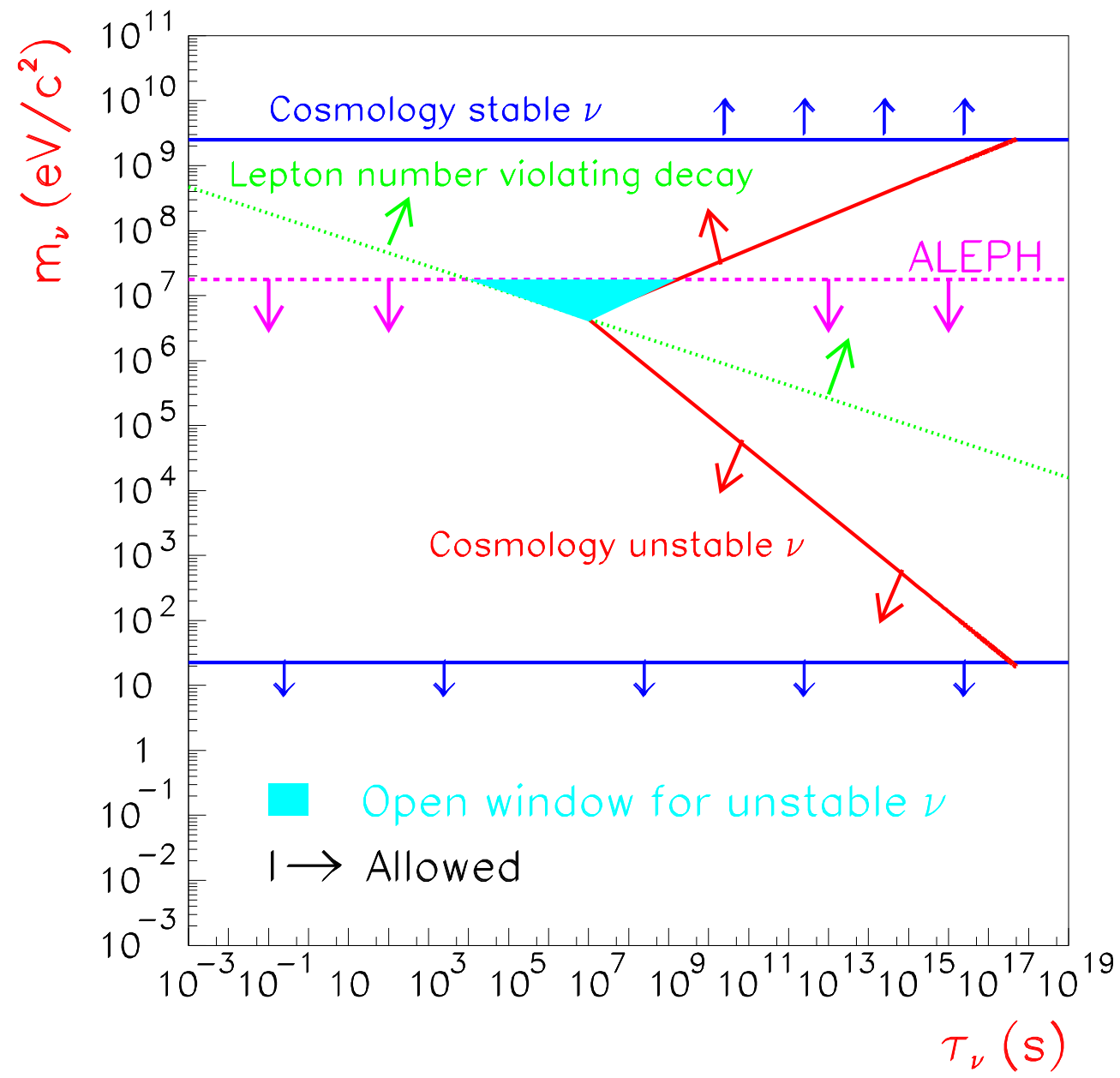

Figure 1: Bounds on $m_{\nu_{\tau}}$ derived from cosmology for a stable or unstable tau neutrino (solid lines). The limits coming from the non-observation of lepton number violating decay (dotted line) and from the direct determination given in this paper (dashed line) are superimposed. The plot is based on the calculation described in Ref. [11]. The gray area shows the allowed region for an unstable neutrino. 


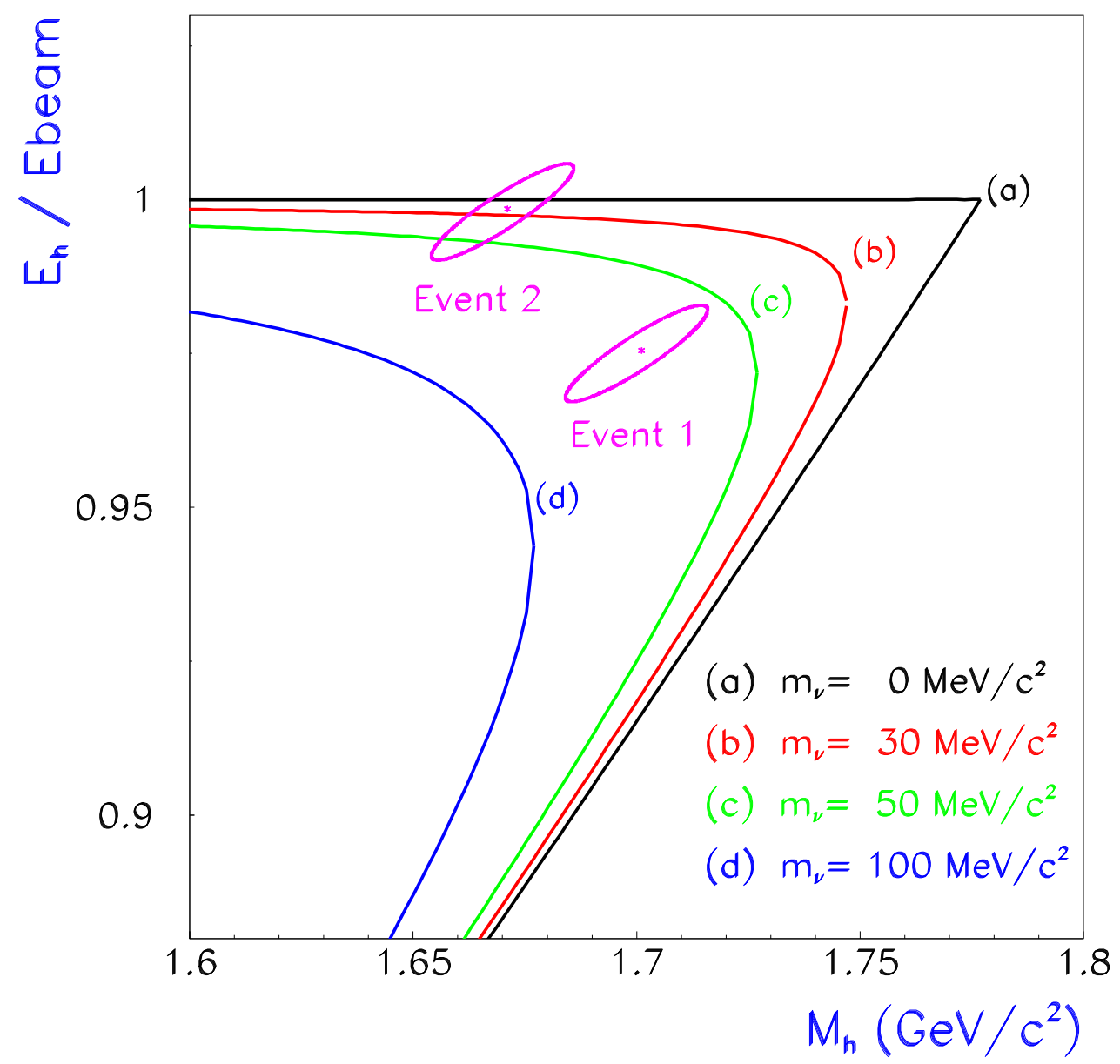

Figure 2: Two hypothetical events with typical $\tau^{-} \rightarrow 2 \pi^{-} \pi^{+} \nu_{\tau}$ error ellipses. The lines indicate the allowed kinematic region for different values of the tau neutrino mass. 


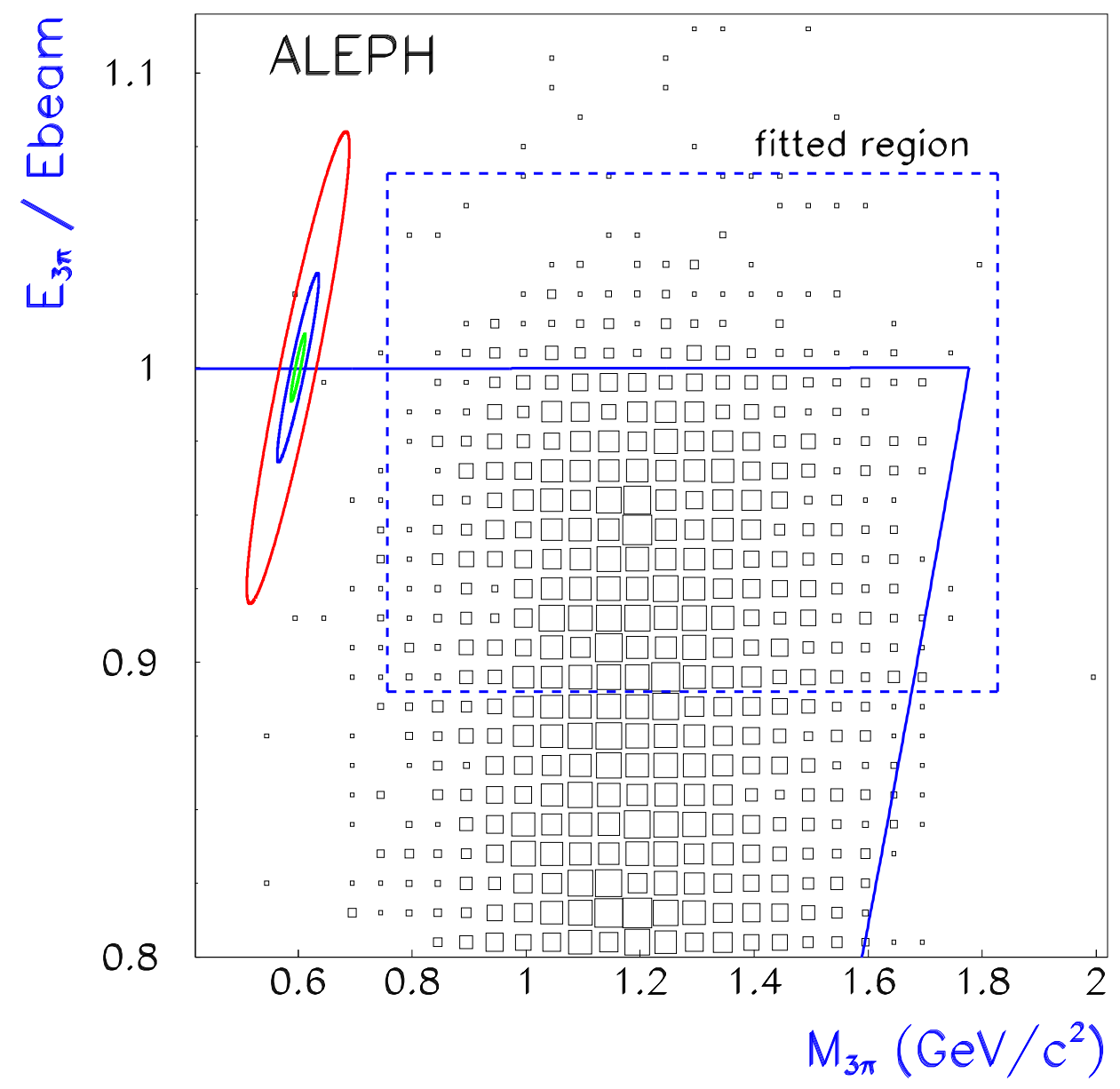

Figure 3: Distribution in the upper part of the $\left(m_{h}, E_{h}\right)$ plane for $\tau^{-} \rightarrow 2 \pi^{-} \pi^{+} \nu_{\tau}$ candidates in the data. The three ellipses at $m_{h}=0.6 \mathrm{GeV} / c^{2}$ show the typical size of the resolution first Gaussian, second Gaussian and the tail. The continuous lines bound the allowed region for a massless neutrino; the dashed box shows the region used in the fit. 


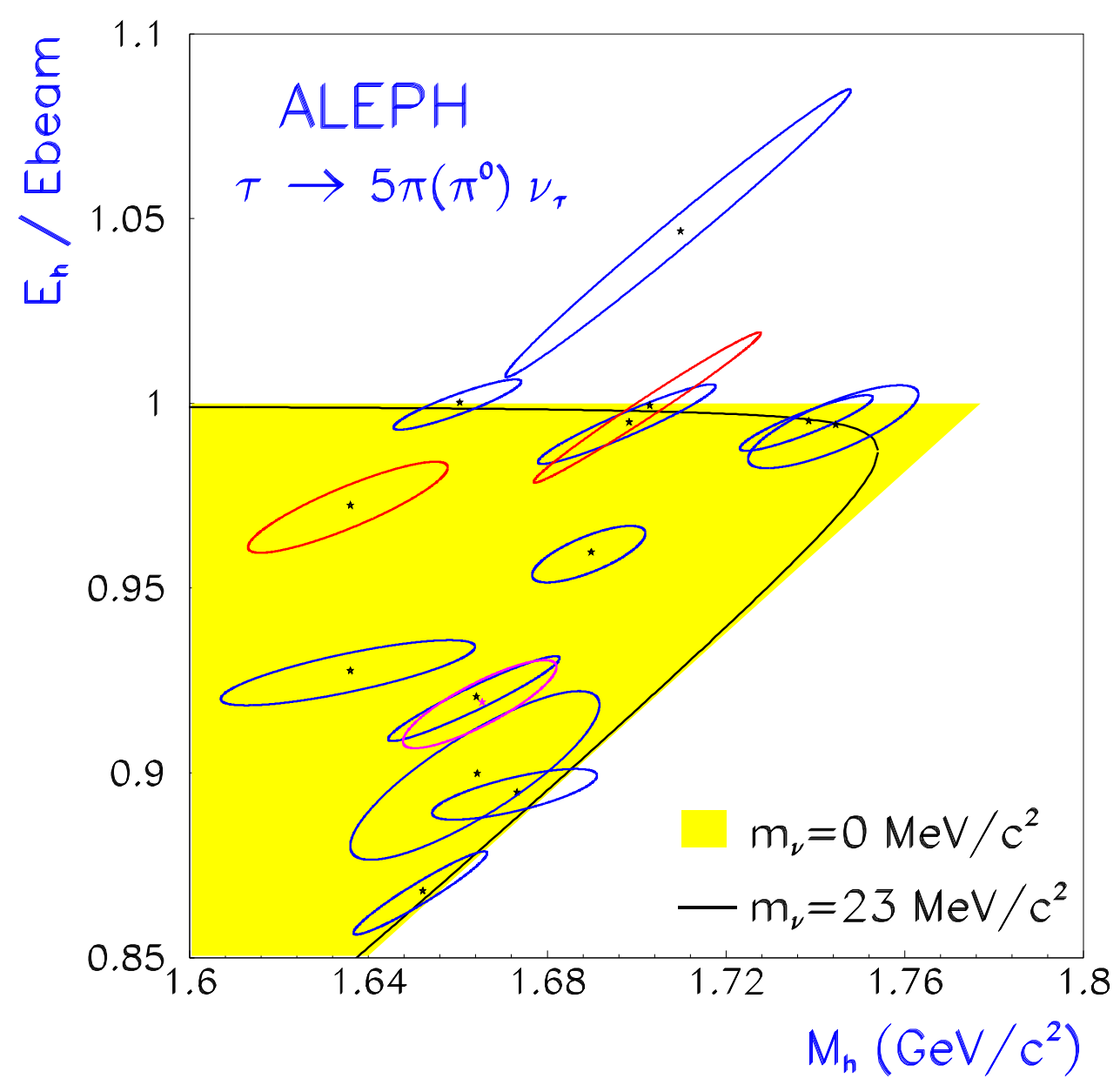

Figure 4: Distribution in the upper part of the $\left(m_{h}, E_{h}\right)$ plane for $\tau^{-} \rightarrow 3 \pi^{-} 2 \pi^{+}\left(\pi^{0}\right) \nu_{\tau}$ candidates in the data. The grey area is the allowed region for a massless neutrino. The borderline of the allowed region for a $23 \mathrm{MeV} / c^{2}$ neutrino is also drawn. The only $\tau^{-} \rightarrow 3 \pi^{-} 2 \pi^{+} \pi^{0} \nu_{\tau}$ event in the plot is the one with the largest hadronic energy. 


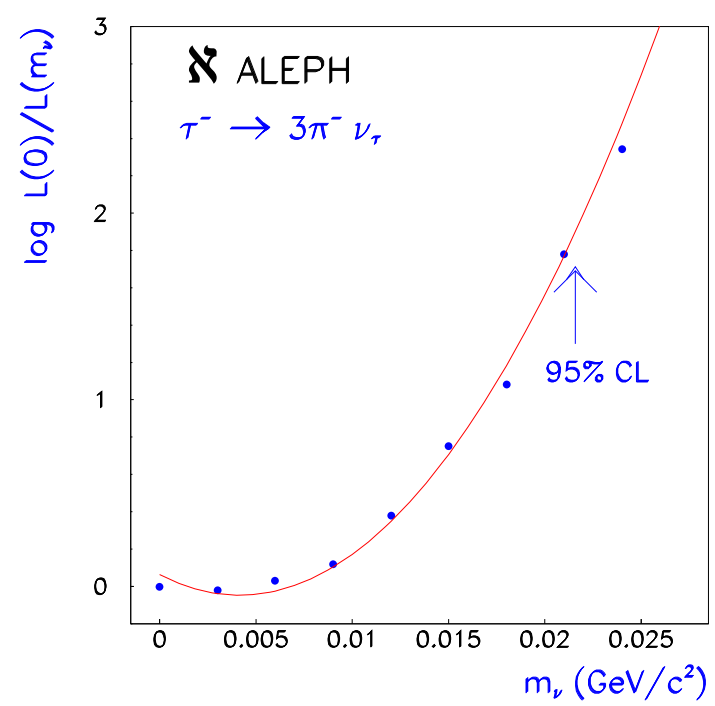

Figure 5: Minus log-likelihood of the $\tau^{-} \rightarrow 2 \pi^{-} \pi^{+} \nu_{\tau}$ data fit as a function of the tau neutrino mass, normalised at $m_{\nu}=0$.

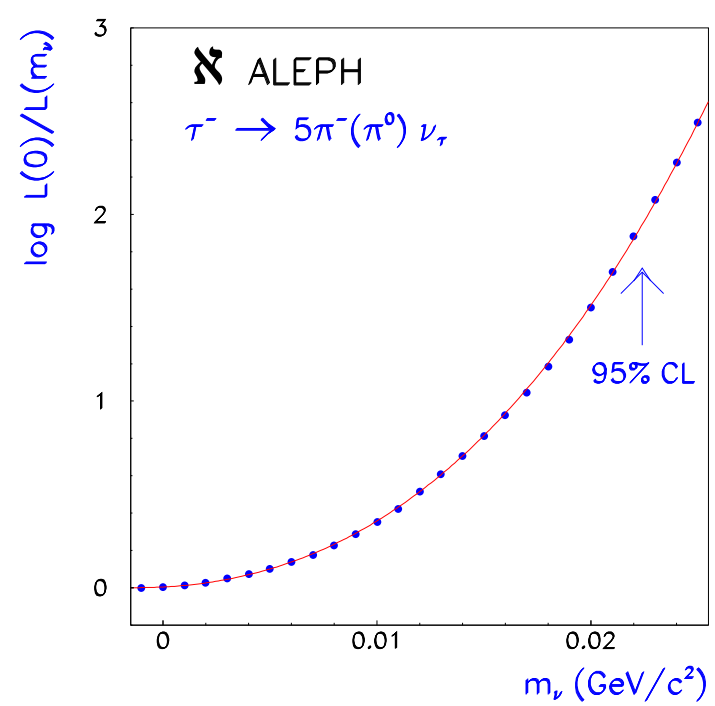

Figure 6: Minus log-likelihood of the $\tau^{-} \rightarrow 3 \pi^{-} 2 \pi^{+}\left(\pi^{0}\right) \nu_{\tau}$ data fit as a function of the tau neutrino mass, normalised at $m_{\nu}=0$. 


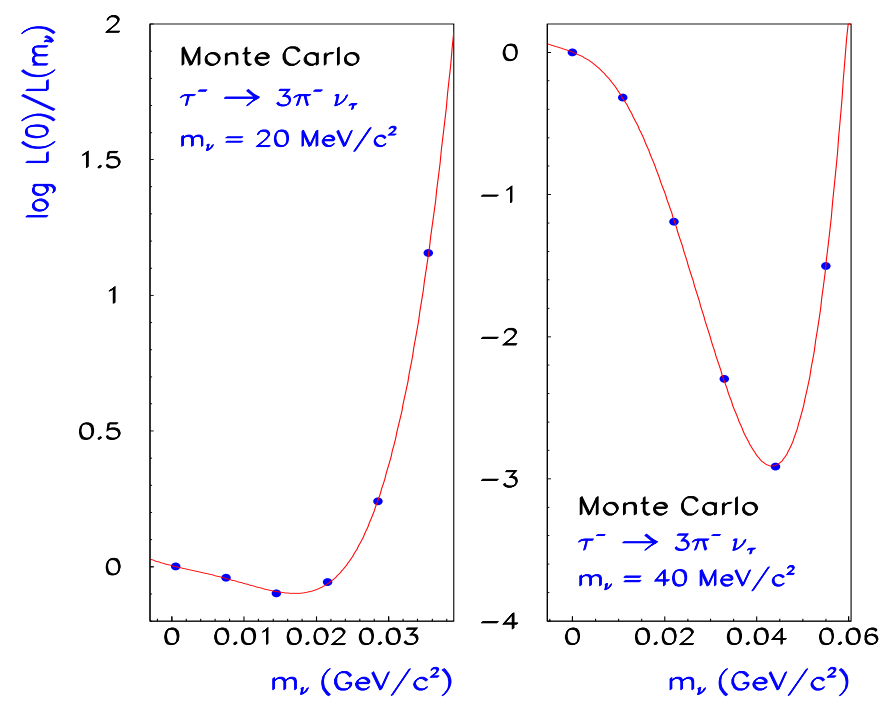

Figure 7: Minus log-likelihood of the $\tau^{-} \rightarrow 2 \pi^{-} \pi^{+} \nu_{\tau}$ fit as a function of the tau neutrino mass normalised at $m_{\nu}=0$ for two Monte Carlo samples with input neutrino masses of 20 and $40 \mathrm{MeV} / c^{2}$. Both samples are statistically equivalent to the data.

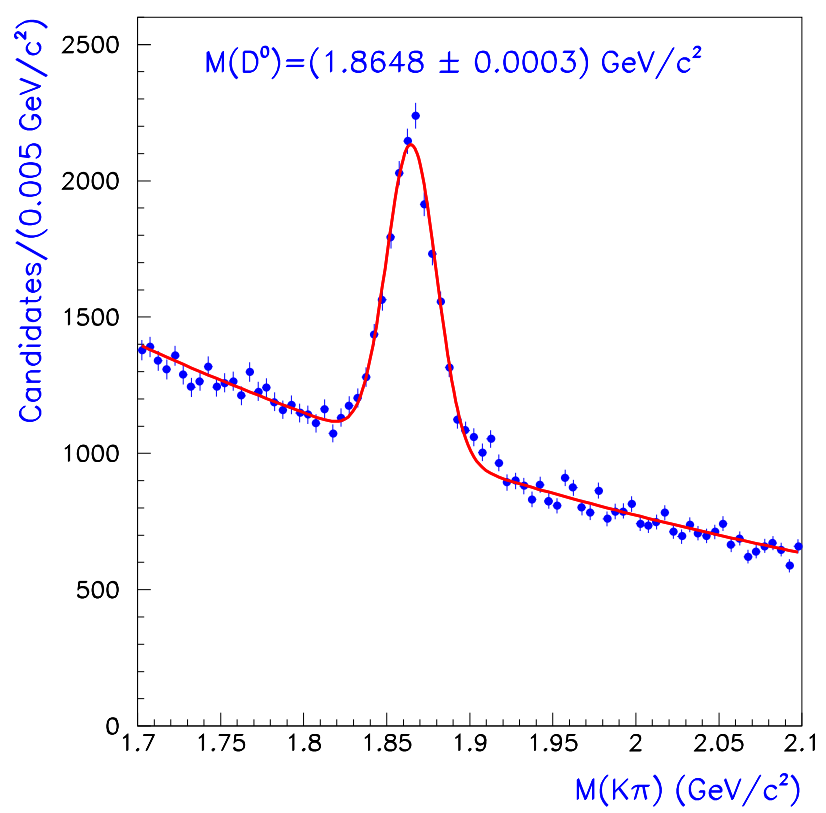

Figure 8: Invariant mass of $D^{0} \rightarrow K^{-} \pi^{+}$data candidates. The result of a fit is superimposed. 


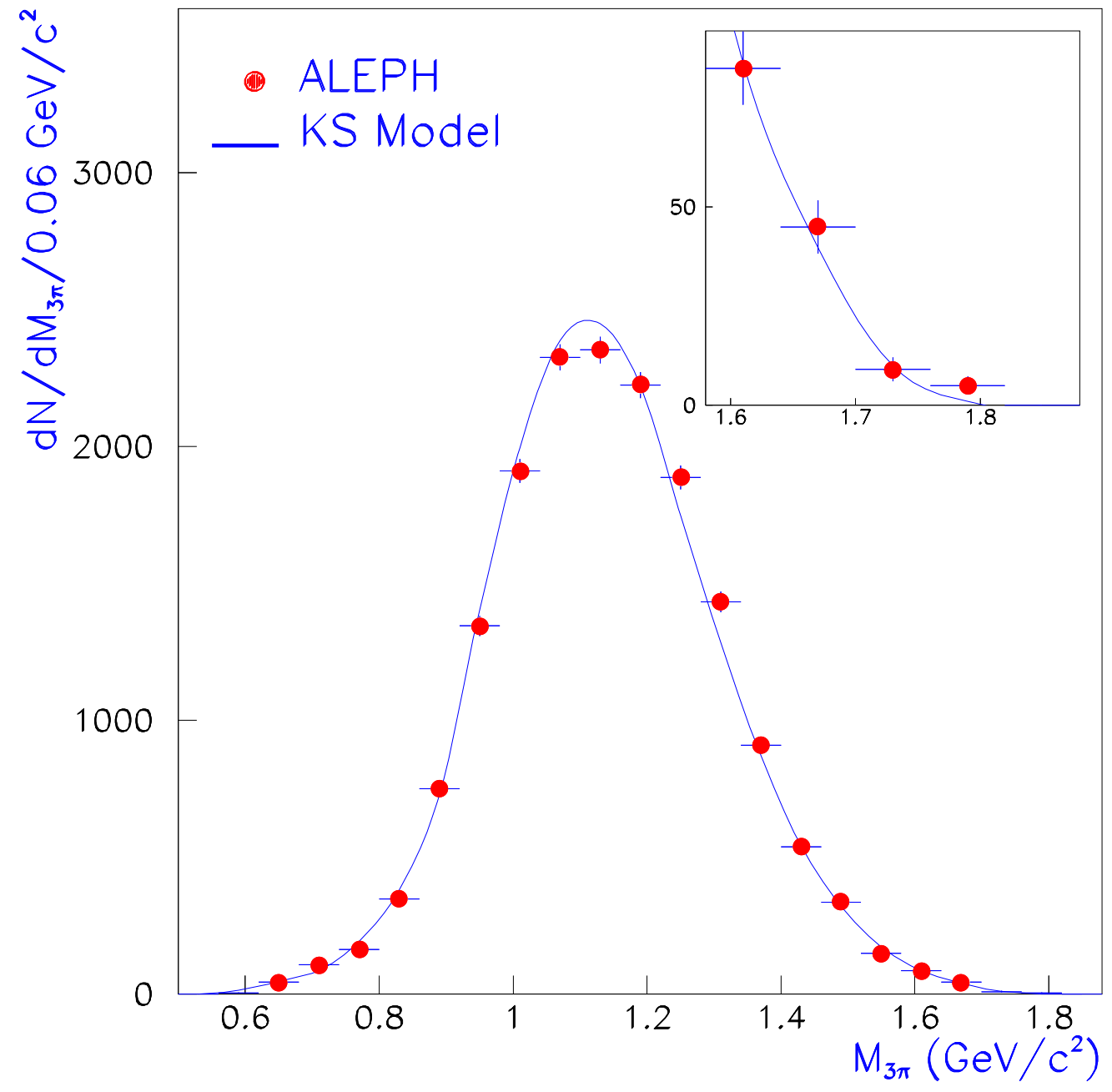

Figure 9: Distribution of $\tau^{-} \rightarrow 2 \pi^{-} \pi^{+} \nu_{\tau}$ invariant mass for data (dots) and the Kühn and Santamaria model (continuous line) for a massless tau neutrino. 


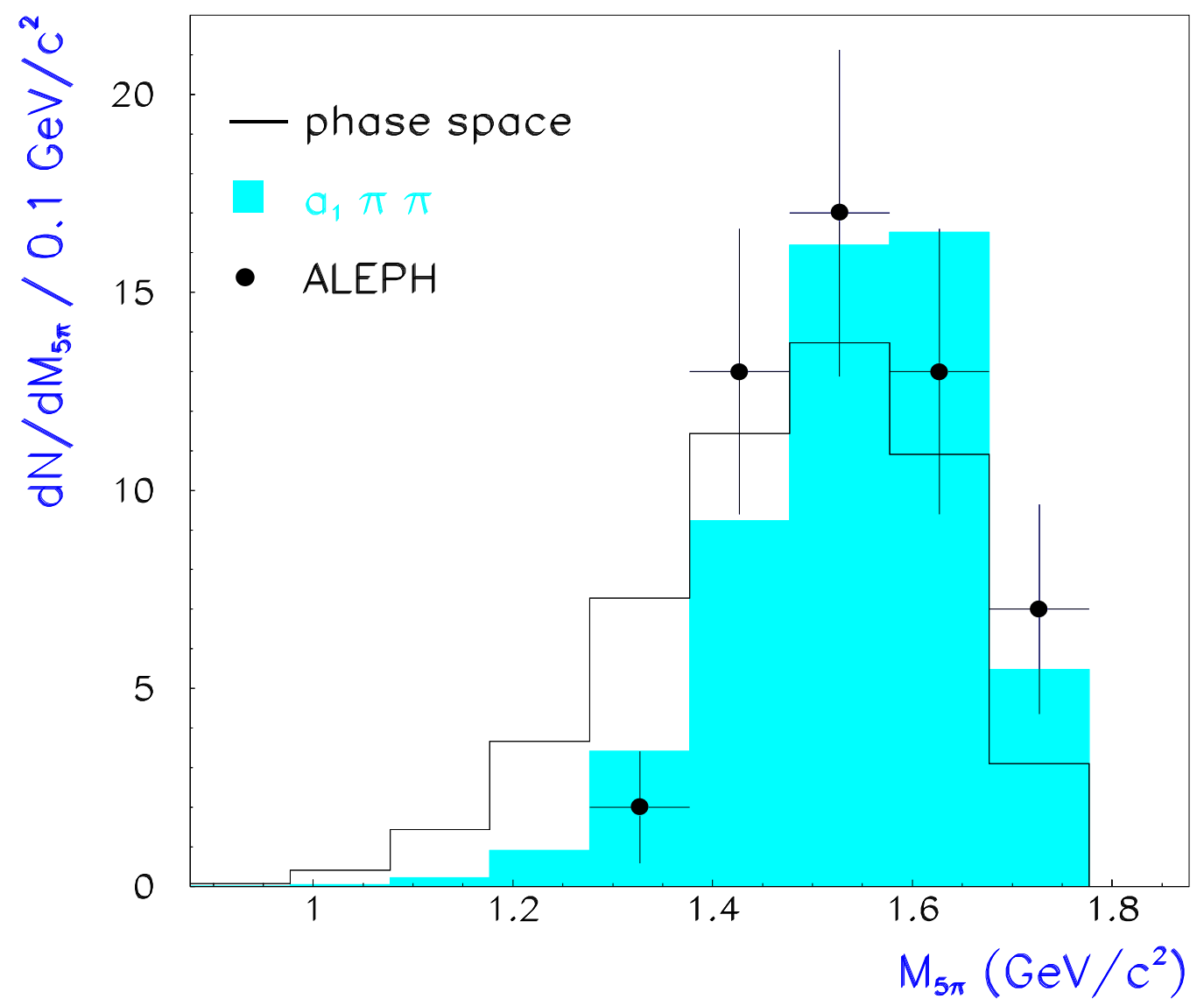

Figure 10: Distribution of $\tau^{-} \rightarrow 3 \pi^{-} 2 \pi^{+} \nu_{\tau}$ invariant mass for data (dots) and two models of decay. The continuous line indicates the pure phase space model while the filled area is obtained by means of an intermediate $a_{1} \pi \pi$ state. 